\title{
Change managen
}

\author{
Veränderungskompetenz entwickeln. Ein Angebot speziell für das Gesundheitswesen
}

Peter Berchtold, College-M

«Change managen» ist ein spezielles, modular aufgebautes Intensivprogramm, das gezielt die nötigen Qualifikationen zur professionellen Gestaltung von Veränderungen im Gesundheitswesen vermittelt.

\section{Ein integrierter Ansatz}

Veränderungen sind heute allgegenwärtig. Sie bieten Chancen, konfrontieren aber auch direkt Personen wie Berufsgruppen und Organisationen im Gesundheitswesen. Keiner bleibt unberührt, niemand kommt darum herum, sich mit ihnen auseinanderzusetzen. Professionelle Veränderungskompetenz ist essentiell geworden.

Professionalität bedeutet sowohl Wissen und Können der relevanten Methoden und Konzepte als auch ein bewusstes Definieren der eigenen Rolle im konkreten Kontext. Diese Anforderungen bedingen die Auseinandersetzung mit der Sache, dem jeweiligen Kontext sowie der eigenen Person. Unser Intensivprogramm fokussiert diesen systemischen Zusammenhang und integriert die wesentlichen Inhalte des Change Managements mit der Arbeit an den kontext- wie personenbezogenen Lerndimensionen.

Diese dichte, für Change Management essentielle Integration von Sache, Kontext und Person zeichnet das Programm besonders aus. Dieser Ansatz erzeugt hohe Effektivität und befähigt die Teilnehmenden, unmittelbar praktisch tätig sein zu können. Unterstützt wird das durch die Arbeit an konkreten Veränderungsprojekten in den Heimatorganisationen der Teilnehmenden. Diese erlaubt eine wesentliche Vertiefung des Lernens durch konkretes Anwenden und Ausprobieren. Lernen erfolgt damit «on the job». Kollegialer Support in Peergruppen sowie projektbezogenes Coaching unterstützen zusätzlich die konkreten Umsetzungen und das Lernen.

\section{Ziele/Inhalte}

- Die Dynamik und Besonderheiten von Veränderungsprozessen verstehen: Was bewegt soziale und ökonomische Systeme? Spezifika der Veränderungen in aktuellen wie zukünftigen Kontexten des Gesundheitssystems.

- Organisationen als Orte der Veränderung begreifen: Organisations- und Führungswissen. Interventionen in Organisationen.

- Veränderungsansätze kennen: Unterschiede, Gemeinsamkeiten, Eignungen im Kontext von Organisationen des Gesundheitswesens.

- Veränderungsprojekte managen: Instrumente, Architekturen, Dynamiken.

- Interventionsstrategien entwickeln: Initiieren, Erarbeiten von Problemanalysen und Lösungssystemen, Implementierungen und Umsetzungen, Gestalten von zielgruppenadäquaten Informations- und Kommunikationsprozessen in Veränderungsprozessen. Strategien in der Unterstützung von einzelnen (Coaching).

- Rolle, Aufträge, und Kontexte: Professionelles Rollenverständnis; Auseinandersetzen mit Erwartungen, Möglichkeiten und Grenzen im Zusammenspiel mit der eigenen Person.

- Konflikte bearbeiten: Interventionsmöglichkeiten und Vorgehen beim Bearbeiten und Lösen von Konflikten (Mediation)

\section{Teilnehmende}

Das Programm richtet sich an Führungspersonen und Projektleiter/innen aus allen Gesundheitsberufen der oberen/mittleren Funktionsebene oder Führungsteams aus Gesundheitsinstitutionen, welche in ihrem beruflichen Umfeld mit Veränderung und Veränderungsprojekten zu tun haben. Die Teilnehmergruppe besteht aus mindestens 12 maximal 18 Personen und soll breit interdisziplinär zusammengesetzt sein.

Die Teilnehmenden bringen ein Projekt bzw. eine Projektidee aus ihrem Arbeitsumfeld mit. Das Projektthema bzw. die Projektidee wird zwischen Programmleitung und Teilnehmenden vereinbart und soll zur institutionellen Verankerung von einem Mentor/einer Mentorin unterstützt werden. 


\section{Programmaufbau und Themen}

Das Programm besteht aus 7 zeitlich getrennten und inhaltlich verknüpften Modulen von total 15 Tagen, zuzüglich 4 halbtägigen Projektcoaching-/Supervisionseinheiten sowie Peergruppenzeit.

Verschiedene Praktiker aus dem Gesundheitswesen werden über erfolgreiche Projekte und Misserfolge berichten.

Ein Eingangsinterview klärt Erwartungen und Ansprüche der Teilnehmenden sowie deren institutionelles Umfeld. Der Aufbau der institutionellen Verankerung kann unterstützt werden.

\section{Arbeitsformen}

Theoretische wie methodische Inputs, Gruppenarbeiten, Rollenspiele, Übungen, Plenums- und Gruppendiskussionen, Eigenreflexionen und fortlaufende Projektarbeiten, unterstützt durch Coaching/Supervision und kollegialen Support, bilden einen methodisch-didaktischen Mix, welcher die Integration der Inhalte und Fragestellungen auf den verschiedenen Lerndimensionen gewährleistet.

\section{Daten}

$\begin{array}{lll}\text { Element 1 } & \text { 4.-6.2.2004 } & \begin{array}{l}\text { Wolfsberg, } \\ \text { Ermatingen TG }\end{array} \\ \text { Element 2 } & \text { 18.-19.3.2004 } & \text { College-M, Bern } \\ \text { Element 3 } & \text { 26.-27.5.2004 } & \text { College-M, Bern } \\ \text { Supervision } & 1.7 .2004 & \text { IEF, Zürich }\end{array}$

Element 4 8.-9.7.2004 College-M, Bern

Supervision 26.8.2004

IEF, Zürich

Element 5 22.-23.9.2004

College-M, Bern

Supervision 28.10.2004 IEF, Zürich

Element 6 18.-19.11.2004 Diakoniewerk, Zollikerberg

Supervision 9.12.2004 IEF Zürich

Element 7 20.-21.1.2005 College-M, Bern

\section{Programmleiter}

- PD Dr. med. Peter Berchtold, College-M, Bern

- Marlies Lenglachner, Corporate Development, Wien

- Mario Patera, Dipl.-Ing. Mag., iff, Wien

- Susanne Quistorp, Dipl.-Psych., Dipl.-Päd., IEF, Zürich

- Dr. Christof Schmitz, Corporate Pragmatics, Zürich

\section{Preis}

Fr. 9660.- (inkl. Kursunterlagen, Übernachtungen: Element 1 [Wolfsberg]

\section{Informationen und Anmeldung}

College-M, Freiburgstrasse 41, 3010 Bern, Tel. 03163230 26, Fax 03163230 25, E-Mail: info@college-m.ch 